\title{
A mutation in the dynein heavy chain gene compensates for energy deficit of mutant SOD1 mice and increases potentially neuroprotective IGF-1
}

Anissa Fergani ${ }^{1,2}$, Judith Eschbach ${ }^{1,2}$, Hugues Oudart ${ }^{2,3}$, Yves Larmet $^{1,2}$, Birgit Schwalenstocker ${ }^{4}$, Albert C Ludolph ${ }^{4}$, Jean-Philippe Loeffler ${ }^{1,2^{*}}$ and Luc Dupuis ${ }^{1,2,4^{*}}$

\begin{abstract}
Background: Amyotrophic lateral sclerosis (ALS) is a fatal neurodegenerative disease characterized by a progressive loss of motor neurons. ALS patients, as well as animal models such as mice overexpressing mutant SOD1s, are characterized by increased energy expenditure. In mice, this hypermetabolism leads to energy deficit and precipitates motor neuron degeneration. Recent studies have shown that mutations in the gene encoding the dynein heavy chain protein are able to extend lifespan of mutant SOD1 mice. It remains unknown whether the protection offered by these dynein mutations relies on a compensation of energy metabolism defects.

Results: SOD1(G93A) mice were crossbred with mice harboring the dynein mutant Cramping allele (Cra/+ mice). Dynein mutation increased adipose stores in compound transgenic mice through increasing carbohydrate oxidation and sparing lipids. Metabolic changes that occurred in double transgenic mice were accompanied by the normalization of the expression of key mRNAs in the white adipose tissue and liver. Furthermore, Dynein Cra mutation rescued decreased post-prandial plasma triglycerides and decreased non esterified fatty acids upon fasting. In SOD1(G93A) mice, the dynein Cra mutation led to increased expression of IGF-1 in the liver, increased systemic IGF-1 and, most importantly, to increased spinal IGF-1 levels that are potentially neuroprotective.

Conclusions: These findings suggest that the protection against SOD1(G93A) offered by the Cramping mutation in the dynein gene is, at least partially, mediated by a reversal in energy deficit and increased IGF-1 availability to motor neurons.
\end{abstract}

\section{Background}

Amyotrophic lateral sclerosis (ALS) is a neurodegenerative disease characterized by a progressive loss of motor neurons in the motor cortex, brainstem and spinal cord. ALS patients develop progressive muscle weakness and paralysis leading to death 3 to 5 years after first symptoms. Despite most cases of ALS occur sporadically, 5\% are genetically inherited. Out of these familial forms of ALS, a subset is caused by mutations in the gene encoding the $\mathrm{Cu} / \mathrm{Zn}$-superoxide dismutase 1 (SOD1).

\footnotetext{
*Correspondence: loeffler@unistra.fr; Idupuis@unistra.fr 'Inserm U692, Laboratoire de Signalisations Moléculaires et Neurodégénérescence, Strasbourg, F-67085 France

Full list of author information is available at the end of the article
}

Studies on patients and transgenic mutant SOD1 mice showed that ALS-linked neurodegeneration was usually associated with defects in energy homeostasis [1]. Indeed, mutant SOD1 mice show a pronounced hypermetabolism characterized by weight loss, increased oxygen consumption and an increased use of lipids stores $[2,3]$. In sporadic ALS patients, hyperlipemia was associated with increased survival $[4,5]$. The mechanisms by which such changes in energy metabolism participate to motor neuron degeneration remain unknown.

Recent studies have shown that mutations in cytoplasmic dynein heavy chain gene protect motor neurons against death and extend survival of mutant SOD1 mice [6-8]. Cytoplasmic dynein is the major molecular motor responsible for retrograde axonal transport in neurons. 
Three different mutations in the dynein heavy chain gene, respectively called "legs at odd angles" (Loa), "Cramping" (Cra) and "Sprawling" (Swl) have been identified in ENU-induced mouse strains $[9,10]$. All three mutations lie in the stem domain of dynein heavy chain and the Loa mutation disrupts, at least partially, the dynein complex [11]. In the nervous system, these mutations lead to perinatal proprioceptive neuropathy [8,10,12]. Cra/+ and Loa/+ mice are hyperactive [13,14], and this is associated, at least in $\mathrm{Cra} /+$ mice with striatal atrophy and compromised neurite outgrowth of striatal neurons [13]. Besides the nervous system, Cra/+ and Loa/+ mice display a major phenotype in adipose tissues [15]. Indeed, Cra/+ and Loa/+ mice show strikingly increased adipose stores, along with compromised thermogenesis. This is most likely due to defective stimulated lipolysis [15].

The extension in lifespan offered by mutations in dynein heavy chain gene has been attributed to several mechanisms, including compensation of axonal transport defects [6] and mitochondrial dysfunction [16], or decreased excitotoxic glutamate input to motor neurons due to degeneration of proprioceptive neurons [8]. In this report we provide evidence that the dynein mutation is able to revert the energy deficit characteristic of ALS in mutant SOD1 mice. Interestingly, this is associated with increased hepatic IGF-1 expression and increased spinal IGF-1. Taking into account these results, we propose that the mutation in dynein is able to provide neuroprotection against SOD1-ALS through complementary pathways.

\section{Results}

Dynein mutation compensates for energy deficit of early symptomatic transgenic SOD1(G93A) mice

We hypothesized that the protection offered by dynein mutation against SOD1(G93A) neurodegeneration was linked to a compensation of the energy deficit of SOD1 (G93A) mice. To test this hypothesis, we crossbred SOD1 (G93A) mice with $\mathrm{Cra} /+$ mice and studied the energetic physiology of compound heterozygotes before any obvious motor symptoms. At 16 weeks of age, SOD1(G93A) nor Cra/SOD1(G93A) mice did not show obvious clinical signs such as gait impairment but both groups of SOD1 (G93A) mice were lighter than wild type littermates. Body weight deficit of Cra/SOD1(G93A) mice was less than SOD1(G93A) mice (not shown) as previously shown [7] and both groups displayed similar upregulation of the denervation marker AchR $\alpha$ in the gastrocnemius muscle compared to non-SOD1(G93A) animals (Figure 1A). This early symptomatic age was selected for further studies. Both groups of SOD1(G93A) mice showed decreased weights of epididymary and retroperitoneal fat pads, but Cra/SOD1(G93A) fat pads were larger than that of SOD1

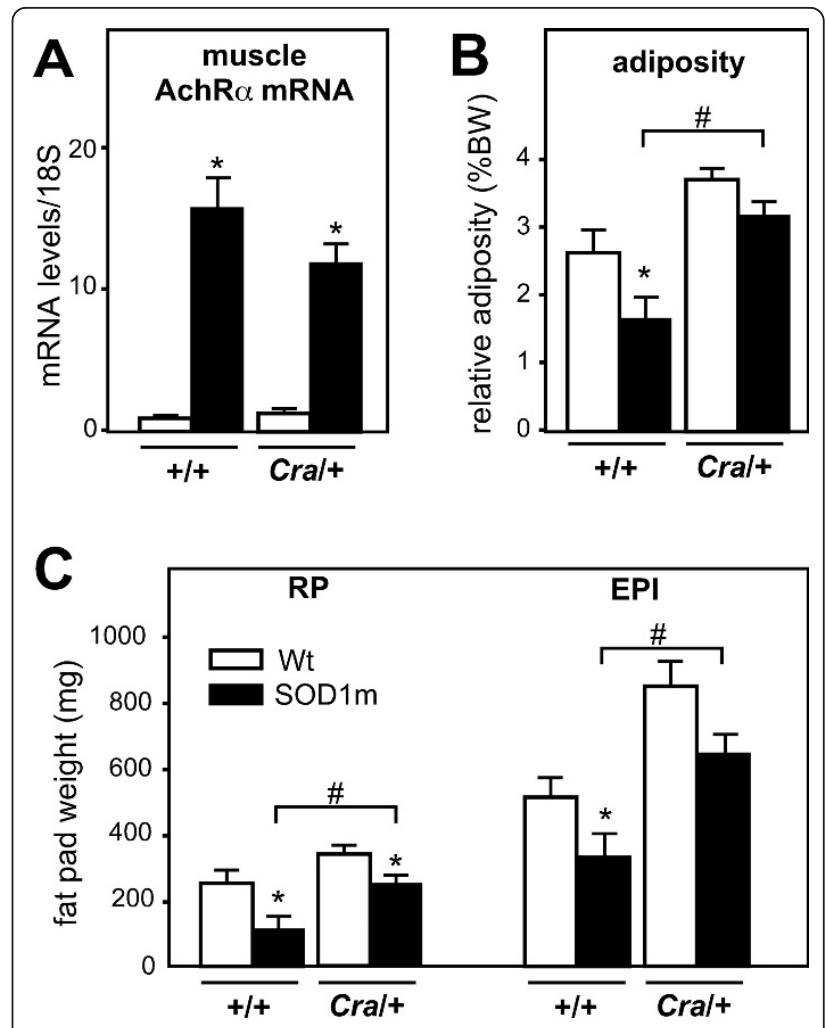

Figure 1 Dynein mutation increased adipose stores in early symptomatic SOD1(G93A) mice. A- mRNA levels of alpha subunit of the nicotinic acetylcholine receptor (AchR $\alpha$ ) in gastrocnemius muscles of wild type $(+/+)$ and dynein mutant mice $(\mathrm{Cra} /+)$ bearing SOD1(G93A) transgene (SOD1m, black columns) or not (Wt, empty columns) $* P<0.05$ versus Wt. mRNA levels were standardized using $18 \mathrm{~S}$ ribosomal RNA as a control. $\mathrm{N}=9$ mice per group. B-C- Relative weight of epididimary (EPI) and retroperitoneal (RP) white adipose tissue fat pad with regard to body weight (B). The panel $C$ shows absolute weight (in $\mathrm{mg}$ ) of $\mathrm{EPI}$ and RP in the same mice than in $\mathrm{A}$. ${ }^{*} P<0.05$ versus $\mathrm{Wt} ; \#, \mathrm{P}<0.05$ as indicated. $\mathrm{N}=9$ mice per group.

(G93A) mice (Figure 1B-C). This was not due to increased food intake since all groups displayed similar food intake (data not shown). In all, these results demonstrate that the Cramping mutation in the dynein heavy chain gene compensated partially for SOD1(G93A) energy deficit in early symptomatic animals.

\section{Dynein mutation does not modify SOD1(G93A) hypermetabolism}

We next determined whether the Cramping dynein mutation compensated for energy deficit through decreased SOD1(G93A) linked hypermetabolism. For this, we measured energy expenditure of the four groups of mice. As previously shown [3], SOD1(G93A) animals had a $15-20 \%$ increase in resting energy expenditure (Figure 2A), and this hypermetabolism was unchanged by Cramping dynein mutation. Furthermore, SOD1(G93A) associated increased total energy expenditure was also 


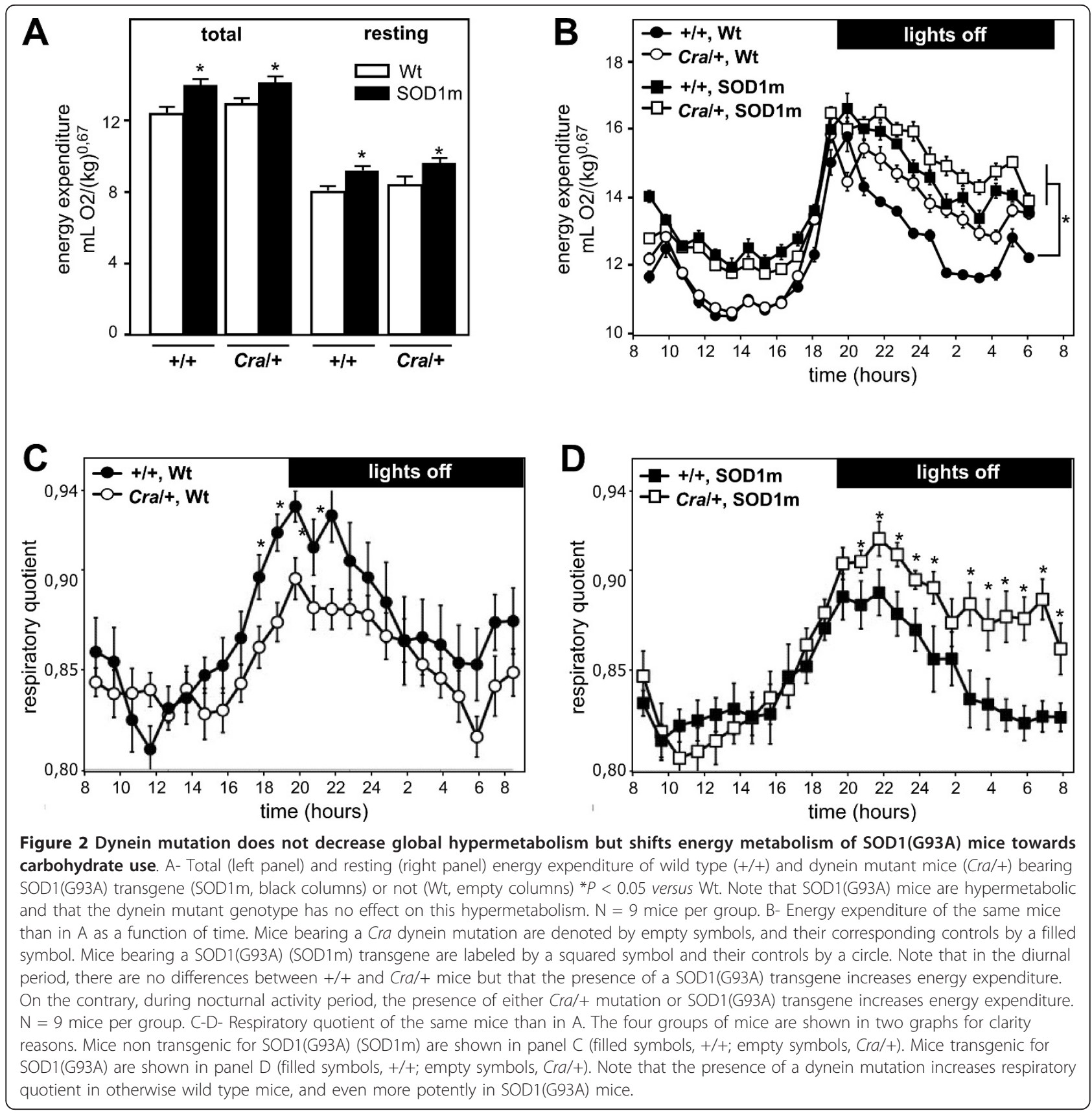

unchanged (Figure 2A) suggesting that the compensation in energy deficit was not provided by, for instance, decreased activity. Thus, the protective potential of Cramping dynein mutation was independent of a direct modulation of global hypermetabolism. Closer examination of indirect calorimetry results revealed however that oxygen consumption of SOD1(G93A) and Cra/SOD1 (G93A) but also $\mathrm{Cra} /+$ mice were increased during the nocturnal period as compared with $+/+$ littermates
(Figure 2B). The respiratory quotient of $\mathrm{Cra} /+$ and $\mathrm{Cral}$ SOD1(G93A) mice was higher than that of $+/+$ and SOD1(G93A) mice at the same period (Figure 2C-D). Since oxidation of carbohydrates leads to a respiratory quotient of 1, while oxidation of lipids leads to a respiratory quotient of 0.7 [17], these results show that the dynein mutation leads to a shift in nutrient use during activity towards preferential carbohydrate oxidation, and thus sparing of lipids and increased fat pad weights. 
Dynein mutation reverts the systemic and molecular changes associated with SOD1(G93A) energy deficit The decreased lipid oxidation in mice bearing the Cramping mutation might be due to their blunted ability to mobilize adipose stores as previously documented [15]. We thus sought to determine whether the increased fat pad weights also reflected a better metabolic status of the mice. Fatty acid synthase (FAS) expression in the liver and Lipoprotein lipase (LPL) expression in white adipose tissue are well known for their regulated expression as a function of nutritional and hormonal cues [18-21]. Most notably, these two genes are profoundly downregulated by energy deficit, including starvation as well as in SOD1(G93A) mice. Here, the Cramping dynein mutation was able to revert these down-regulations in Cra/SOD1(G93A) mice (Figure 3A-B). This increased expression of FAS was associated with unchanged expression of beta-oxidation enzymes such as CPT1A and MCAD or gluconeogenesis enzymes such as PEPCK in the liver (Figure 3B).
However, expression levels of PPAR $\alpha$ and PGC1 $\alpha$, two critical players in the transcriptional control of liver beta-oxidation were decreased by expression of SOD1 (G93A) and expression of PPAR $\gamma$ was increased. The Cramping dynein mutation reverted PPAR $\alpha$ and PGC1 $\alpha$ downregulations and potentiated PPAR $\gamma$ upregulation in Cra/SOD1(G93A) mice (Figure 3B). A hallmark of energy homeostasis defect in SOD1(G93A) mice is the occurrence of decreased circulating triglycerides after feeding [2]. Consistent with these studies, fed, but not fasted; triglycerides were decreased in SOD1(G93A) mice. The Cramping dynein mutation partially compensated for this defect (Figure 3C). Upon fasting, nonesterified fatty acids levels were decreased in SOD1 (G93A) mice, as a likely result of increased muscle uptake $[2,3]$, and this was fully reverted by the Cramping mutation (Figure 3D). The effect of dynein mutation on metabolic gene expression was not observed in skeletal muscle, in which gene expression of MCAD, CPT1B and PGC1 $\alpha$ were unaffected by either SOD1(G93A) or
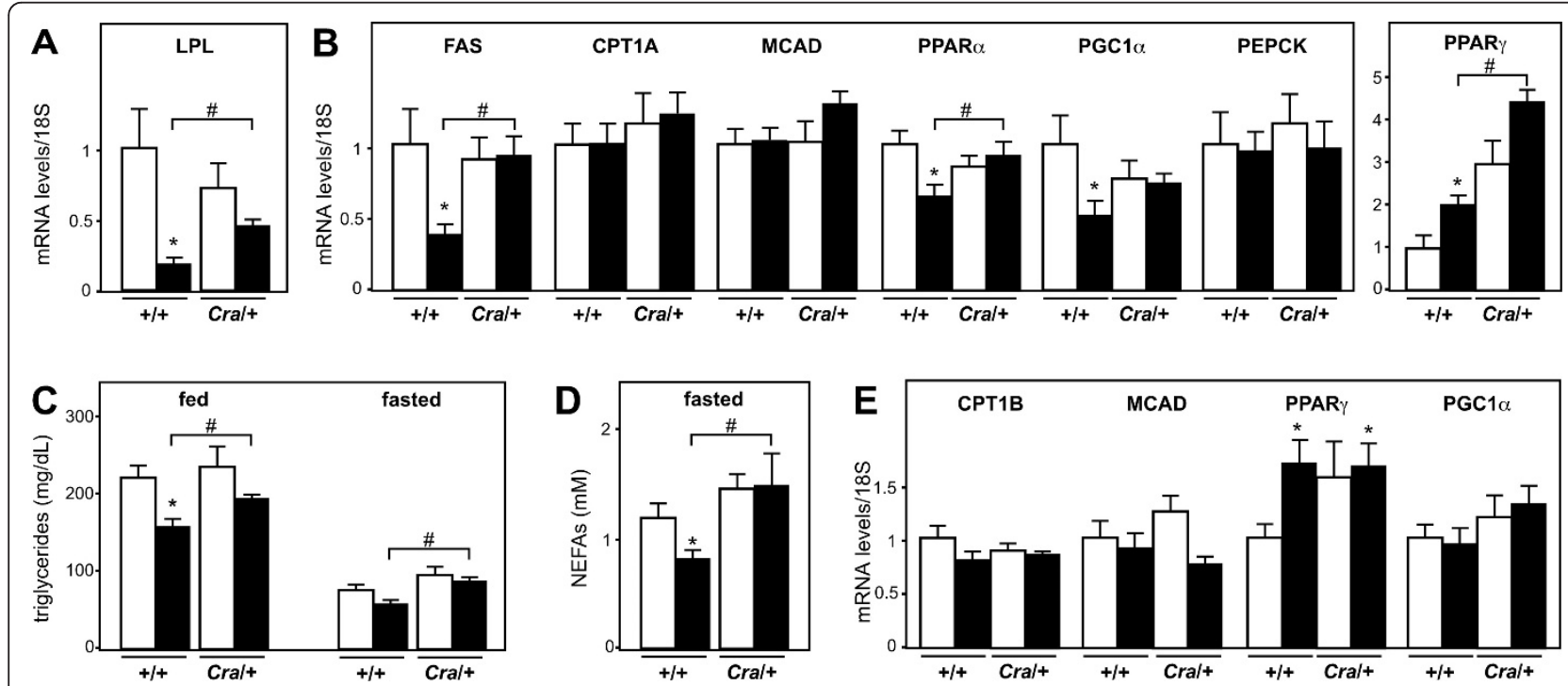

Figure 3 Dynein mutation reverts the systemic and molecular alterations associated with energy deficit in SOD1(G93A) mice. A- mRNA levels of lipoprotein lipase ( $\mathrm{LPL})$ in the epididimary white fat pad of wild type $(+/+)$ and dynein mutant mice (Cra/+) bearing SOD1(G93A) transgene (SOD1m, black columns) or not (Wt, empty columns). ${ }^{*} P<0.05$ versus Wt; $\#, p<0.05$ as indicated. Note that the SOD1(G93A)associated decreased expression of LPL in the EPI is rescued by the dynein mutation in compound SOD1(G93A)/Cra mice. N = 9 mice per group. B- mRNA levels of fatty acid synthase (FAS), carnithine palmitoyl transferase 1A (liver form, CPT1A), Medium chain acyl CoA dehydrogenase (MCAD), peroxisome-proliferator activated receptor alpha (PPAR $\alpha$ ), peroxisome-proliferator activated receptor gamma co-activator 1 alpha (PGC1 $\alpha$ ), phosphoenolpyruvate carboxykinase (PEPCK) and peroxisome-proliferator activated receptor gamma (PPAR $\gamma$ ) in the liver of the same mice than in $\mathrm{A}$. ${ }^{*} P<0.05$ versus $\mathrm{Wt} ; \#, \mathrm{p}<0.05$ as indicated. Note that the SOD1(G93A)-associated decreased expression of FAS in the liver is rescued by the dynein mutation in compound SOD1(G93A)/Cra mice. $\mathrm{N}=9$ mice per group. C-Circulating triglycerides levels in the same mice than in A either in fed (right) or fasted conditions (left). Note that the SOD1(G93A) transgene leads to decreased fed triglycerides levels and that this is partially reverted in compound SOD1(G93A)/Cra mice. $N=9$ mice per group. D- Circulating non-esterified fatty acids (NEFAs) levels in the same mice than in A in fasted conditions. Note that the SOD1(G93A) transgene leads to decreased fasted NEFAs levels and that this is fully reverted in compound SOD1(G93A)/Cra mice. $N=9$ mice per group. E- mRNA levels of carnithine palmitoyl transferase 1B (muscle form, CPT1A), Medium chain acyl CoA dehydrogenase (MCAD), peroxisome-proliferator activated receptor gamma (PPARy) and peroxisome-proliferator activated receptor gamma co-activator 1 alpha $(\mathrm{PGC1} \alpha)$ in the gastrocnemius muscle of the same mice than in $\mathrm{A}$. ${ }^{*} P<0.05$ versus Wt. $\mathrm{N}=9$ mice per group. 
Cramping dynein mutation (Figure 3E). Thus, dynein mutation not only compensates for energy deficit, but also reverts systemic and molecular changes associated with SOD1(G93A) energy deficit.

\section{Dynein mutation increases neuroprotective IGF-1}

We next turned to define potential underlying mechanisms linking energy deficit and motor neuron survival. One candidate mechanism could involve IGF-1. Circulating IGF-1 is a growth factor mainly produced by the liver, but also, in lesser amounts, in other cell types, including muscle, astrocytes and neurons. IGF-1 has been reported to exert neuroprotective effects [22] and to delay motor neuron loss in SOD1(G93A) mice after administration in an early stage of the disease $[23,24]$. IGF-1 expression is known to be decreased upon energy deficit [25]. In SOD1(G93A) mice, liver IGF-1 expression was decreased of about $30 \%$ (Figure $4 \mathrm{~A}$ ), a situation similar to massive energy deficit triggered by $60 \%$ caloric restriction [25]. Consistent with reversal of energy deficit, the Cramping dynein mutation completely reverted IGF-1 mRNA downregulation, and even increased it (Figure 4A). Skeletal muscle IGF-1 mRNA levels were unchanged as were mRNA levels of mechano-growth factor, a muscle specific splice variant of IGF-1 [26] (Figure 4B) suggesting that these changes in IGF-1 mRNA levels were restricted to the liver. We next wanted to determine whether hepatic IGF-1 upregulation translated into increased circulating IGF-1. In SOD1(G93A) mice, plasma IGF-1 levels were surprisingly unchanged, suggesting that other mechanisms, including transcription in other cell types or regulated translation of hepatic IGF-1 mRNA occurred [27]. Plasma IGF-1 levels were modestly increased in Cramping dynein mutant mice but not in Cra/SOD1(G93A) mice (Figure 4C). IGF-1 is poorly but significantly transported throughout the blood brain barrier [28-30] in normal conditions and its entry into the CNS is regulated by neuronal activity [31]. Since the blood brain barrier of SOD1(G93A) mice is disrupted [32,33], it is plausible that an increased fraction of IGF-1 is retained in the spinal cord of Cra/SOD1(G93A) mice. Indeed, spinal IGF-1 was increased in Cramping dynein mutant mice and this was further enhanced by the SOD1 (G93A) transgene (Figure 5A). IGF-1 mRNA levels were unchanged in the spinal cord (Figure 5B) arguing against the proposal that these increased spinal levels of IGF-1 were due to increased local transcription. Last, mRNA levels of MMP-9 were partially restored by the Cramping dynein mutation (Figure 5C) consistent with the hypothesis of increased IGF-1 transcytosis upon dynein mutation. Thus, the dynein mutation increases availability of IGF-1 to motor neurons in the spinal cord. The increased systemic production of IGF-1 by the liver might be the source of increased spinal IGF-1. Such a mechanism could account for the neuroprotective effects of the dynein mutation towards SOD1 (G93A) pathology.

\section{Discussion}

Our current studies provide evidence that the Cramping dynein mutation is able to revert at some point the energy deficit of SOD1(G93A) mice, and to increase IGF-1 levels in the spinal cord of SOD1(G93A) animals.
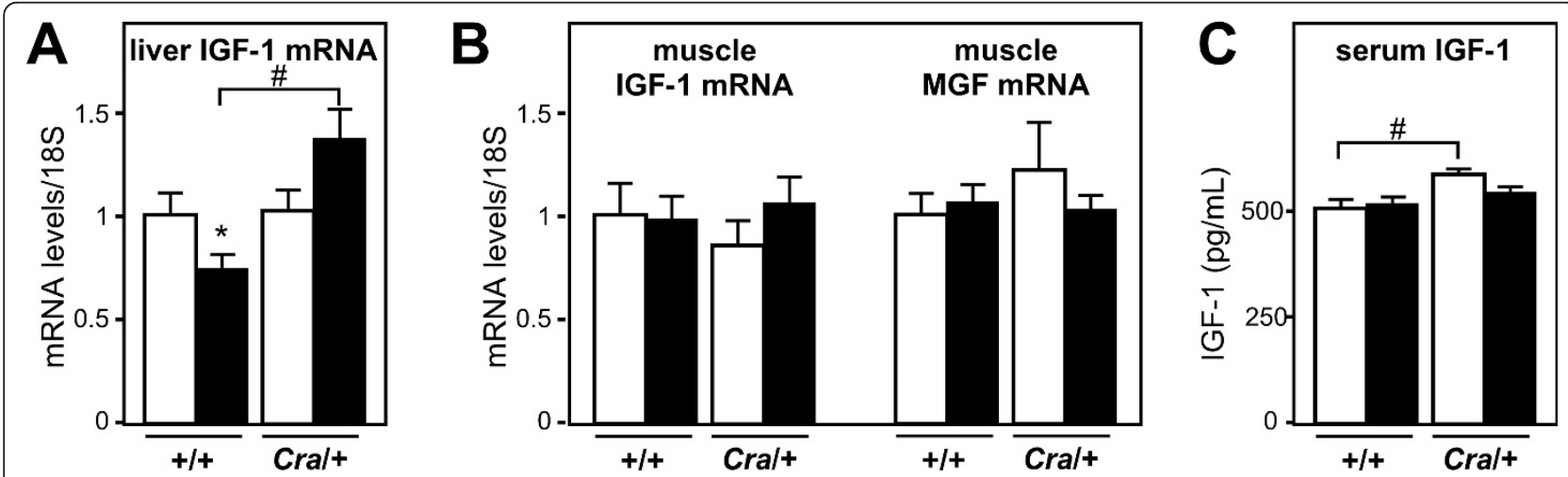

Figure 4 Dynein mutation increases liver IGF-1 expression. A- mRNA levels of Insulin-like growth factor (IGF-1) in the liver of wild type (+/+) and dynein mutant mice (Cra/+) bearing SOD1(G93A) transgene (SOD1m, black columns) or not (Wt, empty columns) ${ }^{*} P<0.05$ versus Wt; \#, $\mathrm{p}$ $<0.05$ as indicated. $N=9$ mice per group. B- mRNA levels of Insulin-like growth factor (IGF-1) and its muscle specific splice variant mechanogrowth factor (MGF) in the gastrocnemius muscle of wild type (+/+) and dynein mutant mice (Cra/+) bearing SOD1(G93A) transgene (SOD1m, black columns) or not (Wt, empty columns). N $=9$ mice per group. C- Serum IGF-1 levels in wild type (+/+) and dynein mutant mice (Cra/+) bearing SOD1(G93A) transgene (SOD1m, black columns) or not (Wt, empty columns) \#, p $<0.05$ as indicated. Note that circulating IGF-1 levels are increased in dynein mutant mice and that this increase is abolished in compound SOD1(G93A)/Cra mice. $N=9$ mice per group. 


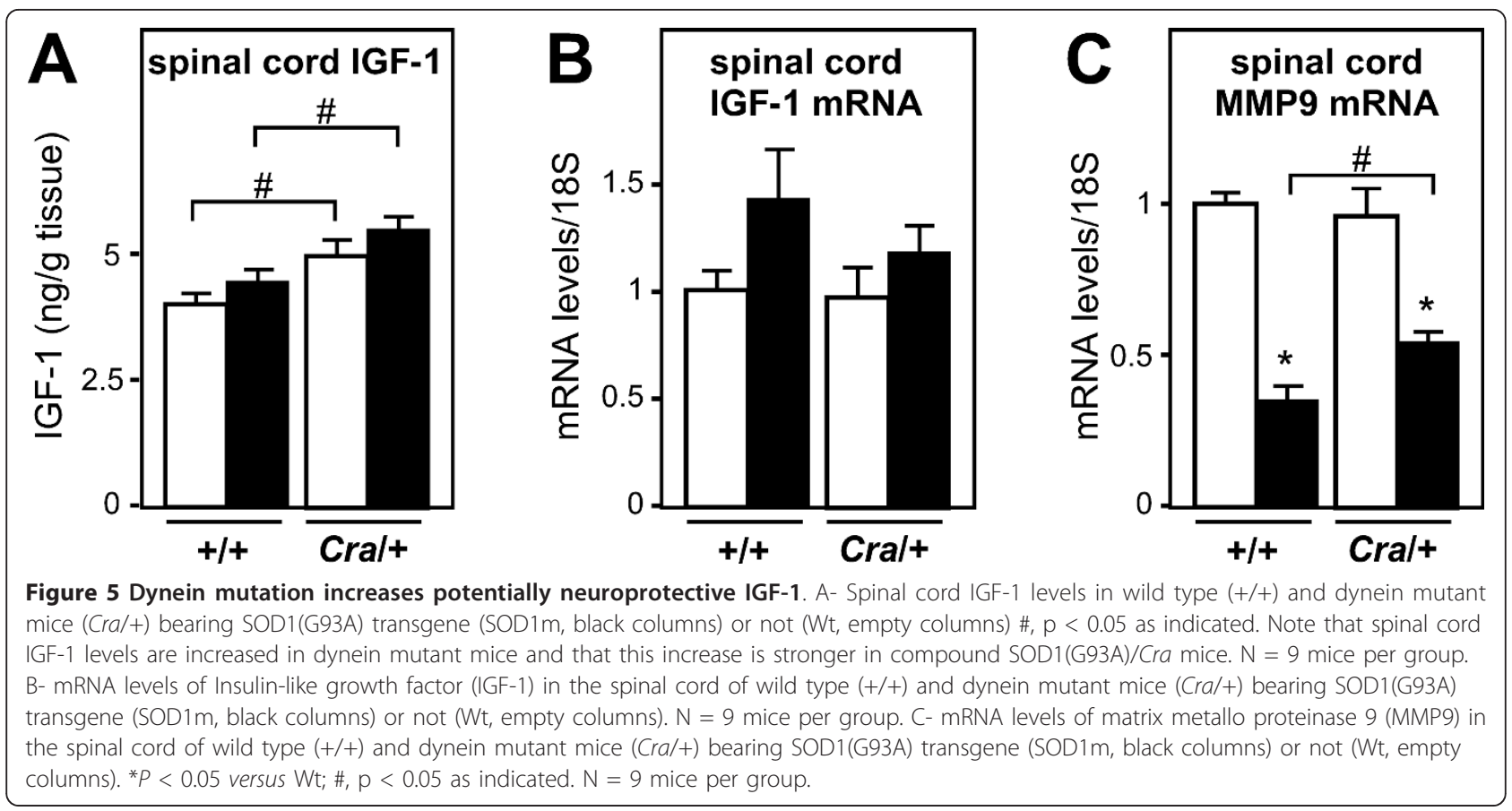

These results, along with previous studies, suggest that two complementary protective pathways are acting in dynein mutant mice to provide the paradoxical protection towards SOD1(G93A) pathology.

Dynein mutation compensates for energy deficit of SOD1 (G93A) mice: a rationale explanation for a seemingly paradoxical observation

This study stems from the seminal observation of a moderate lifespan extension of SOD1(G93A) mice when bearing either Loa or Cra mutation [6-8]. These studies intiated a flurry of research to explain the seemingly paradoxical observation that two distinct molecular injuries that independently lead to ALS-like disease might yield a better outcome than a single one. Indeed, dynein mutant mice were initially thought to develop motor neuron degeneration [9], and it appeared difficult to understand how mutating dynein, and thus precipitating late onset motor neuron degeneration, might protect against early onset SOD1(G93A)-mediated neurodegeneration. Closer examination of dynein mutant mice however refuted that they displayed motor neuron degeneration $[8,10,12]$. In the nervous system, these mice display a frank perinatal sensory neuropathy, accompanied by mild striatal atrophy in the absence of neurodegeneration [13]. Furthermore, apart from this neuronal phenotype, dynein mutant mice also develop a number of peripheral defects, especially in brown and white adipose tissues, that are largely reminiscent of striatal degeneration diseases [15]. The peripheral phenotype of dynein mutant mice was strikingly opposite to that of SOD1(G93A) mice. Indeed, while dynein mutant mice accumulate fats [15], SOD1(G93A) mice are leaner and lose white fat pads with disease progression [3]. The origin of this energy deficit is currently unknown but is associated with increased energy expenditure. Moreover, compensating energy deficit through high fat feeding alleviates motor neuron degeneration [3]. Importantly, these observations are of great relevance for the human pathology since lipemia is positively correlated with survival of ALS patients and energy status appears as a widely documented prognostic factor [1]. In our hands, crossbreeding of dynein mutant mice with SOD1(G93A) mice yielded very similar effects as high fat feeding, by increasing energy storage. Increased RQ during nocturnal period suggests indirectly increased beta-oxidation, while our gene expression analysis are consistent with increased diurnal lipogenesis in compound Cra /SOD1(G93A) mice. Such a metabolic picture is fully consistent with the observed improved energy status. Thus, a straightforward interpretation would be that the dynein mutant mediated injury compensates mutant SOD1 injury through its effect on energy homeostasis, thereby alleviating neurodegeneration.

\section{Potential involvement of IGF-1 in dynein mutant mediated protection}

We further provide mechanistic insights into how the dynein mutant peripheral phenotype might provide protection to motor neurons. Liver IGF-1 expression is under the control of nutritional cues, and is for instance decreased in starved animals [25]. Consistent with their 
energetic status, we observed downregulation of IGF-1 in SOD1(G93A) liver, and this was fully reverted by dynein mutation. It should be noted however that SOD1 (G93A) showed only limited analogy with starved animals since liver expression of genes such as PPAR $\alpha$ or PGC1 $\alpha$ showed regulations opposite to those observed in starved animals. In double mutant mice, while there were no changes in circulating IGF-1, we observed increased spinal IGF-1. A similar trend was observed between wild type and SOD1(G93A) animals. One may consider that the increased hepatic production of IGF-1, due to the reversal of the energy deficit of SOD1(G93A) mice, is directed towards the nervous system, and thus motor neurons, of SOD1(G93A) mice. This is consistent with our results at the different levels at which we investigated IGF-1 (liver, skeletal muscle, plasma and spinal cord). This interpretation is further in line with modified expression of IGF-1 receptors and IGF-1 binding proteins that occur in SOD1(G93A) mice motor neurons $[34,35]$. It should be noted here that circulating, liver derived IGF-1 is able to cross the blood brain barrier (BBB) and counteract age-related decline in cognitive functions $[36,37]$. This natural entry might even be potentiated by leakage of the BBB during ALS disease [32,33]. Also, IGF-1 transcytosis has been shown to be dependent upon neuronal activity, which leads to increased MMP-9 activation and cleavage of IGF binding proteins [31]. Interestingly, we previously observed increased activity of MMP-9 in SOD1(G93A) spinal cord [38] and others have documented decreased levels of IGF binding proteins [35] in SOD1(G93A) CNS. Decreased MMP9 mRNA levels in sick SOD1(G93A) mice is not at odds with previous results since we and others have shown that MMP9 activity actually peaks before onset and then decreases [38,39]. In all, our results support that IGF-1 transcytosis might be increased in SOD1(G93A), and to an even greater extent in $\mathrm{Cra} / \mathrm{SOD} 1$ (G93A) mice.

Our study does not directly address whether increased IGF-1 is responsible for the extension in lifespan. This is however plausible as most studies observed protective effect of increasing IGF-1 in SOD1 (G93A) mice [23,24]. A direct positive impact on neuromuscular junctions from circulating IGF-1 cannot be excluded since muscle-restricted expression of IGF-1 is able to stabilize neuromuscular junctions and delay motor neurons death of SOD1(G93A) G93A mice [24]. Also, IGF-1 is able to provide protection to mitochondria even at low doses in aging rats $[40,41]$ through a boost in mitochondrial biogenesis and our results are thus consistent with the recent observation of mitochondrial protection in SOD1(G93A) mice by dynein mutation [16]. In human clinical trials however, IGF-1 did not achieve efficacy in ALS clinical trials [42]. This lack of efficacy might be due to inappropriate targeting of IGF-1 to motor neurons (see below). Alternatively, IGF-1 might be acting in combination with other still unknown circulating factors to provide full protection in dynein mutant mice. In all, we propose that circulating factors modified by the reversal of energy deficit, and between these, IGF-1, contribute to the protection offered by dynein mutation.

\section{Mutant dynein mediated protection may rest on two complementary biological events}

Several research groups, including ours, have previously shown that dynein Cra and Loa mutations were able to increase the lifespan of SOD1G93A mice. Several mechanisms have been proposed to explain the underlying mechanisms of mutant dynein protection towards mutant SOD1 pathogenesis. To conclude, we would like here to discuss critically these different hypotheses in the light of our results and provide a working model summarizing these different potential mechanisms.

In the first study, Kieran et al. [6] suggested a cell autonomous effect of dynein mutation in motor neurons and proposed that the defect in retrograde transport triggered by mutant dynein counterbalanced the one due to SOD1(G93A) in the anterograde direction, and thereby restoring axonal homeostasis. This cell autonomous protective effect of dynein mutation is in line with results obtained by Teuling and collaborators [43] that used neuron-specific overexpression of an $\mathrm{N}$-terminal deleted form of BicD2 to modulate dynein/cargo interaction [44]. Interestingly, BicD2 overexpression in neurons disrupted retrograde axonal transport, delayed the SOD1 aggregates retrograde transport toward the cell center and increased survival of SOD1 G93A mice [43]. However, BicD2 overexpression might also have a number of dynein independent effects, including on kinesinmediated transport [45] Pan-neuronal overexpression of BicD2 might also lead to impairment of neuronal physiology in neurons other than motor neurons, including in proprioceptive neurons (see below). Thus, the hypothesis of the restoration of axonal homeostasis by double blockade of both anterograde and retrograde directions of axonal transport remains unproven.

The complex phenotype of dynein mutant mice render very plausible that dynein mutation could also act at multiple levels besides motor neurons. First, dynein mutation is likely to reduce glutamate excitotoxicity through the degeneration of proprioceptive glutamate inputs to motor neurons [8]. Although this hypothesis is initially attractive, type Ia afferents that degenerate in dynein mutant mice stimulate also inhibitory interneurons to perform reciprocal inhibition [46]. The exact effect of dynein mutation on excitotoxic load on motor neurons thus remains to be investigated. Besides 


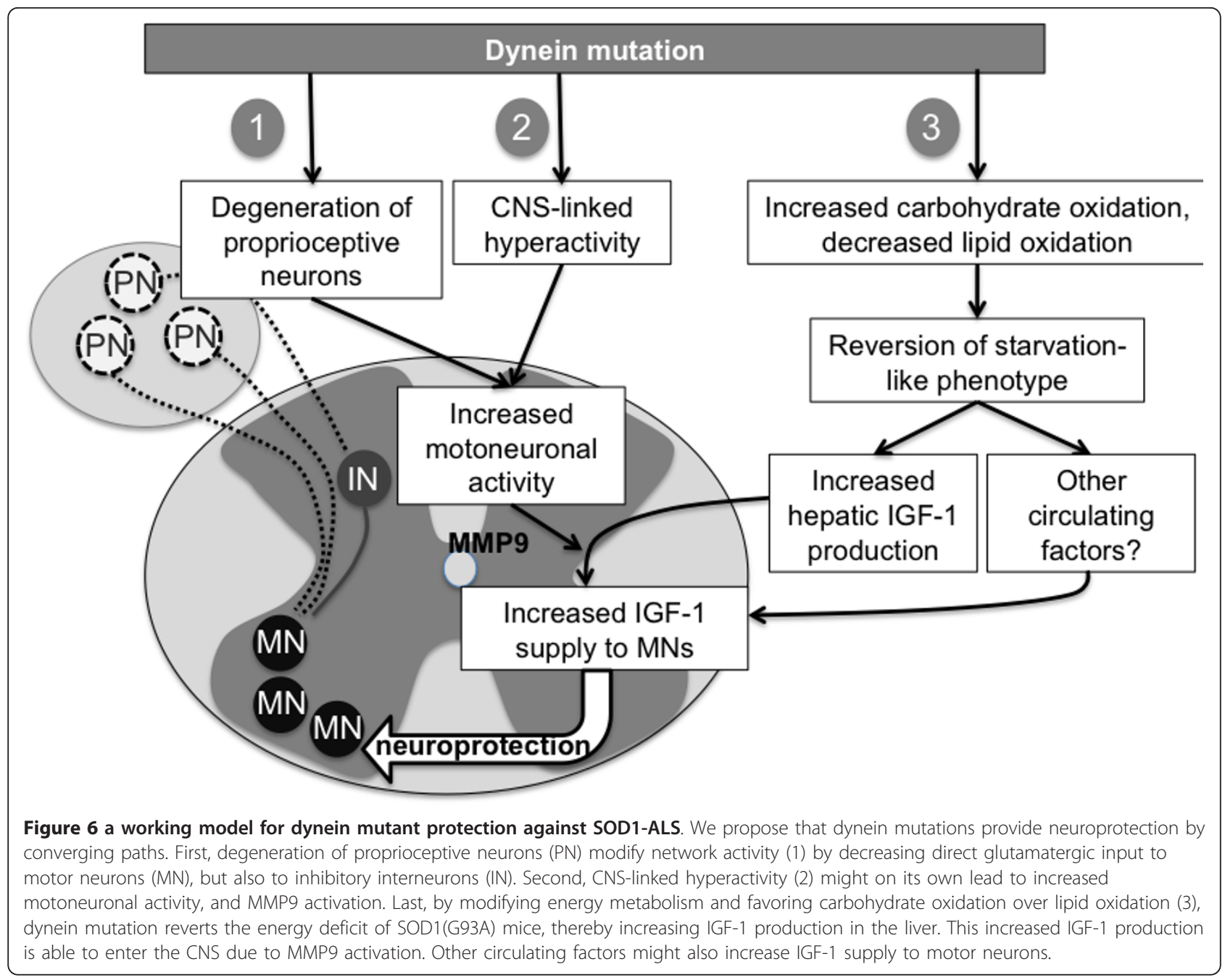

proprioceptive afferents, motoneuronal activity is likely to be increased in dynein mutant mice due to increased spontaneous activity [13]. Our current findings lead us to propose that the protective dynein mutations, such as Legs at odd angles and Cramping, also impact bioenergetics' mechanisms and revert the energy deficit of SOD1(G93A) mice. In the compound mice, this leads to increased production of IGF-1. We propose that the proprioceptive degeneration and CNS linked hyperactivity converge to modify neuronal activity and facilitate the net transcytosis of IGF-1 in the vicinity of motor neurons, thus achieving neuroprotection. This working model is summarized in Figure 6.

\section{Conclusions}

These findings suggest that the protection against SOD1 (G93A) offered by the Cramping mutation in the dynein gene is, at least partially, mediated by a reversal in energy deficit and increased IGF-1 availability to motor neurons. Our study provides a rationale explanation to increased survival in double dynein mutant/SOD1 (G93A) mice and suggests that the neuroprotection results from dynein mutant phenotype in various tissues.

\section{Methods}

Animals

Heterozygous $\mathrm{Cra} /+$ females were crossed with SOD93A males and were identified by tail DNA genotyping for the human transgene SOD1 (G93A) and the Cra mutation as described previously [7,9]. Experiments were performed with littermates mice with 9 mice per group. Mice were maintained at $23^{\circ} \mathrm{C}$ with a $12 \mathrm{~h}$ light/dark cycle and had food and water ad libitum. All animal experiments were performed under the supervision of authorized investigators and followed current EU regulations. Animals were treated in accordance with the European Union guide for the care and the use of animals in research (commission June 18th 2007, 2007/526/CE). 
LD has been allowed to perform mouse experiments (agreement A67-266, direction des services vétérinaires, Strasbourg, Bas-Rhin, France).

\section{Indirect calorimetry}

We measured $\mathrm{O}_{2}$ consumption and $\mathrm{CO}_{2}$ production by using an open-circuit indirect calorimetry system (Sable systems, Las Vegas, USA). Concentrations of $\mathrm{O}_{2}$ and $\mathrm{CO}_{2}$ in the outgoing air were successively measured in five different cages. The system was rinsed for $90 \mathrm{~s}$ between each measurement. Final values of gas concentrations were the mean of 10 measures obtained during $40 \mathrm{~s}$. Each cage was sampled every $11 \mathrm{~min}$, and one cage was left vacant as reference of ambient gas concentrations. Measurements were performed continuously over $231 / 2 \mathrm{~h}$, a 30-min period being required for calibration of the $\mathrm{O}_{2}$ and $\mathrm{CO}_{2}$ analyzers. In total, 127 measures were collected per day and mouse. The average of the five lowest values of $\mathrm{O}_{2}$ consumption was considered as resting energy expenditure. Energy expenditure was obtained by using an energy equivalent of $20.1 \mathrm{~J} / \mathrm{ml}$ $\mathrm{O}_{2}$. The respiratory quotient was the ratio of $\mathrm{CO}_{2}$ production over $\mathrm{O}_{2}$ consumption.

\section{RT-qPCR}

RNA were extracted by using Trizol $^{\circledR}$ (Invitrogen, Cergy-Pontoise, France). Real time RT quantitative PCR was performed with one microgramme of total RNA as described [13]. PCR analysis was carried out on a BioRad iCycler System using iQSYBR Green Supermix. A specific standard curve was performed in parallel for each gene, and each sample was quantified in duplicate. PCR conditions were $3 \mathrm{~min}$ at $94^{\circ} \mathrm{C}$, followed by 40 cycles of $45 \mathrm{~s}$ at $94^{\circ} \mathrm{C}$ and $10 \mathrm{~s}$ at $60^{\circ} \mathrm{C}$. The relative levels of each RNA were normalized to 18S RNA levels. Oligonucleotide sequences are reported in the table 1.

\section{Biochemical assays}

Mouse IGF-1 levels were measured using Mouse/Rat IGF-I Quantikine ELISA Kit (R\&D systems), using manufacturer's instructions. Plasma triglycerides and NEFAs

\section{Table 1 Oligonucleotides sequences}

\begin{tabular}{lll}
\hline $\begin{array}{l}\text { Name of } \\
\text { the gene }\end{array}$ & Forward primer & Reverse primer \\
\hline 18S & TCTGATAAATGCACGCATCC & GCCATGCATGTCTAAGTACGC \\
AchRalpha & CCACAGACTCAGGGGAGAAG & AACGGTGGTGGTGTGATG \\
CPT1a & GCTGTCAAAGATACCGTGAGC & TCTCCCTCCTTCATCAGTGG \\
CPT1b & TGCCTITACATCGTCTCCAA & GGCTCCAGGGTCAGAAAGT \\
FAS & TCTGCAGAGAAGCGAGCATA & CCCAGAGGGTGTTGTAGA \\
IGF-1 & GCTTGCTCACCTTACCAGC & AAATGTACTTCCTTCTGGTCT \\
MCAD & TGTCGAACACAACACTCGAAA & CTGCTGTTCCGTCAACTCAA \\
\hline
\end{tabular}

were measured using Randox kits using manufacturer's instructions.

\section{Statistical Analysis}

Statistical comparisons were accomplished with the unpaired Student $t$ test, unless otherwise indicated, or ANOVA followed by the post hoc Newman-Keuls multiple comparisons test using PRISM version 2.0a software (GraphPad, San Diego).

\section{List of abbreviations}

AchRa: alpha subunit of the nicotinic cholinergic receptor; ALS: amyotrophic lateral sclerosis; BBB: blood brain barrier; BicD2: bicaudal D2; CNS: central nervous system; Cra: Cramping mutation of the Dyn1hc1 gene; FAS: fatty acid synthase; IGF-1: Insulin-like growth factor 1; LPL: lipoprotein lipase; SOD1: copper-zinc superoxide dismutase.

\section{Acknowledgements}

This work was supported by grants from Fondation pour la Recherche Médicale, Association pour la Recherche sur la Sclérose Latérale Amyotrophique (A.R.S.) and Amyotrophic lateral sclerosis association (grant 1698) to L.D.; and Association Française contre les Myopathies, A.R.S. and Association pour la Recherche et le Développement de Moyens de Lutte contre les Maladies Neurodégénératives to J.P.L and by Deutsche Forschungsgemeinschaft (KFO142) to ACL. We acknowledge the skilful technical assistance of Marie-José Ruivo, Olivier Schandène and Annie Picchinenna. LD is supported by a grant from the "agence nationale de la recherche" (ANR) (young researcher program, Dynemit).

\section{Author details}

${ }^{1}$ Inserm U692, Laboratoire de Signalisations Moléculaires et Neurodégénérescence, Strasbourg, F-67085 France. ²Université de Strasbourg, Faculté de Médecine, UMRS692, Strasbourg, F-67085 France. ${ }^{3}$ DEPE, IPHC, Strasbourg, France. ${ }^{4}$ Department of Neurology, University of Ulm, Ulm, Germany.

\section{Authors' contributions}

AF performed animal handling and follow up, participated in the indirect calorimetry experiments, performed qPCR assays and most of the biochemical assays; JE performed GPCR assays and some biochemical assays, analyzed indirect calorimetry results and drafted the manuscript; $\mathrm{HO}$ performed indirect calorimetry experiments; $Y L$ performed animal follow up and analyzed results; BS performed animal handing, genotyping and analyzed results; $A C L$ and JPL conceived of the study, participated in its design and helped to draft the manuscript; LD conceived of the study, designed and coordinated the study and drafted the manuscript. All authors read and approved the final manuscript.

\section{Competing interests}

The authors declare that they have no competing interests.

Received: 2 December 2010 Accepted: 26 April 2011

Published: 26 April 2011

\section{References}

1. Dupuis L, Pradat PF, Ludolph AC, Loeffler JP: Energy metabolism in amyotrophic lateral sclerosis. Lancet Neurol 2010, 10(1):75-82.

2. Fergani $A$, Oudart $H$, Gonzalez De Aguilar JL, Fricker B, Rene F, Hocquette JF, Meininger $V$, Dupuis L, Loeffler JP: Increased peripheral lipid clearance in an animal model of amyotrophic lateral sclerosis. J Lipid Res 2007, 48:1571-1580.

3. Dupuis L, Oudart H, Rene F, Gonzalez de Aguilar JL, Loeffler JP: Evidence for defective energy homeostasis in amyotrophic lateral sclerosis: benefit of a high-energy diet in a transgenic mouse model. Proc Natl Acad Sci USA 2004, 101:11159-11164.

4. Dupuis L, Corcia P, Fergani A, Gonzalez De Aguilar JL, BonnefontRousselot D, Bittar R, Seilhean D, Hauw JJ, Lacomblez L, Loeffler JP, 
Meininger $\mathrm{V}$ : Dyslipidemia is a protective factor in amyotrophic lateral sclerosis. Neurology 2008, 70:1004-1009.

5. Dorst J, Kühnlein P, Hendrich C, Kassubek J, Sperfeld AD, Ludolph AC Patients with elevated triglyceride and cholesterol serum levels have a prolonged survival in Amyotrophic Lateral Sclerosis. I Neurol 2010, 258(4):613-7.

6. Kieran D, Hafezparast M, Bohnert S, Dick JR, Martin J, Schiavo G, Fisher EM Greensmith L: A mutation in dynein rescues axonal transport defects and extends the life span of ALS mice. J Cell Biol 2005, 169:561-567.

7. Teuchert M, Fischer D, Schwalenstoecker B, Habisch HJ, Bockers TM, Ludolph AC: A dynein mutation attenuates motor neuron degeneration in SOD1(G93A) mice. Exp Neurol 2006, 198:271-274.

8. Ilieva HS, Yamanaka K, Malkmus S, Kakinohana O, Yaksh T, Marsala M, Cleveland DW: Mutant dynein (Loa) triggers proprioceptive axon loss that extends survival only in the SOD1 ALS model with highest motor neuron death. Proc Natl Acad Sci USA 2008, 105:12599-12604.

9. Hafezparast M, Klocke R, Ruhrberg C, Marquardt A, Ahmad-Annuar A, Bowen S, Lalli G, Witherden AS, Hummerich H, Nicholson S, et al: Mutations in dynein link motor neuron degeneration to defects in retrograde transport. Science 2003, 300:808-812.

10. Chen XJ, Levedakou EN, Millen KJ, Wollmann RL, Soliven B, Popko B: Proprioceptive sensory neuropathy in mice with a mutation in the cytoplasmic Dynein heavy chain 1 gene. J Neurosci 2007, 27:14515-14524.

11. Deng W, Garrett C, Dombert B, Soura V, Banks G, Fisher EM, van der Brug MP, Hafezparast M: Neurodegeneration mutation in cytoplasmic dynein alters its organization and dynein-dynactin and dynein-kinesin interactions. J Biol Chem 2010.

12. Dupuis L, Fergani A, Braunstein KE, Eschbach J, Holl N, Rene F, Gonzalez De Aguilar JL, Zoerner B, Schwalenstocker B, Ludolph AC, Loeffler JP: Mice with a mutation in the dynein heavy chain 1 gene display sensory neuropathy but lack motor neuron disease. Exp Neurol 2009, 215:146-152.

13. Braunstein KE, Eschbach J, Rona-Voros K, Soylu R, Mikrouli E, Larmet $Y$, Rene F, Gonzalez De Aguilar JL, Loeffler JP, Muller HP, et al: A point mutation in the dynein heavy chain gene leads to striatal atrophy and compromises neurite outgrowth of striatal neurons. Hum Mol Genet 2010

14. Rogers DC, Peters J, Martin JE, Ball S, Nicholson SJ, Witherden AS, Hafezparast M, Latcham J, Robinson TL, Quilter CA, Fisher EM: SHIRPA, a protocol for behavioral assessment: validation for longitudinal study of neurological dysfunction in mice. Neurosci Lett 2001, 306:89-92.

15. Eschbach J, Fergani A, Oudart $H$, Robin JP, Rene F, de Aguilar JL, Larmet $Y$, Zoll J, Hafezparast M, Schwalenstocker B, et al: Mutations in cytoplasmic dynein lead to a Huntington's disease-like defect in energy metabolism of brown and white adipose tissues. Biochim Biophys Acta 2010, 1812(1):59-69.

16. El-Kadi AM, Bros-Facer V, Deng W, Philpott A, Stoddart E, Banks G Jackson GS, Fisher EM, Duchen MR, Greensmith L, et al: The legs at odd angles (Loa) mutation in cytoplasmic dynein ameliorates mitochondrial function in SOD1G93A mouse model for motor neuron disease. $J$ Biol Chem 2010, 285:18627-18639.

17. Livesey G, Elia M: Estimation of energy expenditure, net carbohydrate utilization, and net fat oxidation and synthesis by indirect calorimetry: evaluation of errors with special reference to the detailed composition of fuels. Am J Clin Nutr 1988, 47:608-628.

18. Laux T, Schweizer M: Dietary-induced pre-translational control of rat fatty acid synthase. Biochem J 1990, 266:793-797.

19. Kim TS, Freake HC: High carbohydrate diet and starvation regulate lipogenic mRNA in rats in a tissue-specific manner. J Nutr 1996, 126:611-617.

20. Sugden MC, Holness MJ, Howard RM: Changes in lipoprotein lipase activities in adipose tissue, heart and skeletal muscle during continuous or interrupted feeding. Biochem J 1993, 292(Pt 1):113-119.

21. Galan X, Llobera M, Ramirez I: Lipoprotein lipase and hepatic lipase in Wistar and Sprague-Dawley rat tissues. Differences in the effects of gender and fasting. Lipids 1994, 29:333-336.

22. Vincent AM, Mobley BC, Hiller A, Feldman EL: IGF-I prevents glutamateinduced motor neuron programmed cell death. Neurobiol Dis 2004, 16:407-416.

23. Kaspar BK, Llado J, Sherkat N, Rothstein JD, Gage FH: Retrograde viral delivery of IGF-1 prolongs survival in a mouse ALS model. Science 2003, 301:839-842.
24. Dobrowolny G, Giacinti C, Pelosi L, Nicoletti C, Winn N, Barberi L, Molinaro M, Rosenthal N, Musaro A: Muscle expression of a local lgf-1 isoform protects motor neurons in an ALS mouse model. I Cell Biol 2005, 168:193-199.

25. Oster MH, Fielder PJ, Levin N, Cronin MJ: Adaptation of the growth hormone and insulin-like growth factor-I axis to chronic and severe calorie or protein malnutrition. J Clin Invest 1995, 95:2258-2265.

26. Matheny RW, Nindl BC, Adamo ML: Minireview: Mechano-growth factor: a putative product of IGF-I gene expression involved in tissue repair and regeneration. Endocrinology 2010, 151:865-875.

27. Lee EK, Gorospe M: Minireview: posttranscriptional regulation of the insulin and insulin-like growth factor systems. Endocrinology 2010, 151:1403-1408.

28. Pan W, Kastin AJ: Interactions of IGF-1 with the blood-brain barrier in vivo and in situ. Neuroendocrinology 2000, 72:171-178.

29. Armstrong CS, Wuarin L, Ishii DN: Uptake of circulating insulin-like growth factor-I into the cerebrospinal fluid of normal and diabetic rats and normalization of IGF-II mRNA content in diabetic rat brain. J Neurosci Res 2000, 59:649-660.

30. Reinhardt RR, Bondy CA: Insulin-like growth factors cross the blood-brain barrier. Endocrinology 1994, 135:1753-1761.

31. Nishijima T, Piriz J, Duflot S, Fernandez AM, Gaitan G, Gomez-Pinedo U, Verdugo JM, Leroy F, Soya H, Nunez A, Torres-Aleman I: Neuronal activity drives localized blood-brain-barrier transport of serum insulin-like growth factor-I into the CNS. Neuron 2010, 67:834-846.

32. Garbuzova-Davis S, Saporta S, Haller E, Kolomey I, Bennett SP, Potter H, Sanberg PR: Evidence of compromised blood-spinal cord barrier in early and late symptomatic SOD1 mice modeling ALS. PLOS One 2007, 2:e1205.

33. Zhong Z, Deane R, Ali Z, Parisi M, Shapovalov Y, O'Banion MK, Stojanovic K, Sagare A, Boillee S, Cleveland DW, Zlokovic BV: ALS-causing SOD1 mutants generate vascular changes prior to motor neuron degeneration. Nat Neurosci 2008, 11:420-422.

34. Narai H, Nagano I, llieva H, Shiote M, Nagata T, Hayashi T, Shoji M, Abe K: Prevention of spinal motor neuron death by insulin-like growth factor-1 associating with the signal transduction systems in SODG93A transgenic mice. J Neurosci Res 2005, 82:452-457.

35. Sim SE, Chung YH, Jeong JH, Yun SW, Lim HS, Kim D, Kim SS, Lee WB, Cha Cl: Immunohistochemical localization of insulin-like growth factor binding protein 2 in the central nervous system of SOD1(G93A) transgenic mice. J Mol Histol 2009, 40:157-163.

36. Svensson J, Diez M, Engel J, Wass C, Tivesten A, Jansson JO, Isaksson O, Archer T, Hokfelt T, Ohlsson C: Endocrine, liver-derived IGF-I is of importance for spatial learning and memory in old mice. J Endocrinol 2006, 189:617-627.

37. Trejo JL, Piriz J, Llorens-Martin MV, Fernandez AM, Bolos M, LeRoith D, Nunez A, Torres-Aleman I: Central actions of liver-derived insulin-like growth factor I underlying its pro-cognitive effects. Mol Psychiatry 2007, 12:1118-1128

38. Fang L, Teuchert M, Huber-Abel F, Schattauer D, Hendrich C, Dorst J, Zettlmeissel H, Wlaschek M, Scharffetter-Kochanek K, Kapfer T, et al: MMP-2 and MMP-9 are elevated in spinal cord and skin in a mouse model of ALS. J Neurol Sci 2010, 294:51-56.

39. Soon CP, Crouch PJ, Turner BJ, McLean CA, Laughton KM, Atkin JD, Masters CL, White AR, Li QX: Serum matrix metalloproteinase-9 activity is dysregulated with disease progression in the mutant SOD1 transgenic mice. Neuromuscul Disord 2010, 20:260-266.

40. Puche JE, Garcia-Fernandez M, Muntane J, Rioja J, Gonzalez-Baron S, Castilla Cortazar I: Low doses of insulin-like growth factor-I induce mitochondrial protection in aging rats. Endocrinology 2008, 149:2620-2627.

41. Echave P, Machado-da-Silva G, Arkell RS, Duchen MR, Jacobson J, Mitter R, Lloyd AC: Extracellular growth factors and mitogens cooperate to drive mitochondrial biogenesis. J Cell Sci 2009, 122:4516-4525.

42. Sorenson EJ, Windbank AJ, Mandrekar JN, Bamlet WR, Appel SH, Armon C, Barkhaus PE, Bosch P, Boylan K, David WS, et al: Subcutaneous IGF-1 is not beneficial in 2-year ALS trial. Neurology 2008, 71:1770-1775.

43. Teuling E, van Dis V, Wulf PS, Haasdijk ED, Akhmanova A, Hoogenraad CC, Jaarsma D: A novel mouse model with impaired dynein/dynactin function develops amyotrophic lateral sclerosis (ALS)-like features in motor neurons and improves lifespan in SOD1-ALS mice. Hum Mol Genet 2008, 17:2849-2862. 
44. Dienstbier M, Li X: Bicaudal-D and its role in cargo sorting by microtubule-based motors. Biochem Soc Trans 2009, 37:1066-1071

45. Splinter D, Tanenbaum ME, Lindqvist A, Jaarsma D, Flotho A, Yu KL, Grigoriev I, Engelsma D, Haasdijk ED, Keijzer N, et al: Bicaudal D2, dynein, and kinesin-1 associate with nuclear pore complexes and regulate centrosome and nuclear positioning during mitotic entry. PLOS Biol 2010, 8:e1000350.

46. Kandel ER, Schwartz JH, Jessell TM: Principles of neural science. 4 edition. McGraw-Hill, New York; 2000

47. Dupuis L, Gonzalez de Aguilar JL, Echaniz-Laguna A, Eschbach J, Rene F, Oudart H, Halter B, Huze C, Schaeffer L, Bouillaud F, Loeffler JP: Muscle mitochondrial uncoupling dismantles neuromuscular junction and triggers distal degeneration of motor neurons. PLoS One 2009, 4:e5390.

doi:10.1186/1750-1326-6-26

Cite this article as: Fergani et al.: A mutation in the dynein heavy chain gene compensates for energy deficit of mutant SOD1 mice and increases potentially neuroprotective IGF-1. Molecular Neurodegeneration $20116: 26$

\section{Submit your next manuscript to BioMed Central} and take full advantage of:

- Convenient online submission

- Thorough peer review

- No space constraints or color figure charges

- Immediate publication on acceptance

- Inclusion in PubMed, CAS, Scopus and Google Scholar

- Research which is freely available for redistribution

Submit your manuscript at www.biomedcentral.com/submit 